\title{
Foraminiferal stratigraphy in a marine Eemian- Weichselian sequence at Apholm, North Jutland
}

\author{
KAREN LUISE KNUDSEN
}

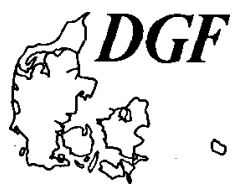

Knudsen, K. L.: Foraminiferal stratigraphy in a marine Eemian-Weichselian sequence at Apholm, North Jutland. Bull. geol. Soc. Denmark, vol. 32, pp. 169-180, Copenhagen, September, 4th, 1984. https://doi.org/10.37570/bgsd-1983-32-13

\begin{abstract}
Undisturbed core material is investigated for the first time from a marine Quatemary sequence through the Eemian of North Jutland. Core samples were examined from $86 \mathrm{~m}-190 \mathrm{~m}$ depth in a $202.6 \mathrm{~m}$ deep boring at Apholm. The marine sequence from that interval was subdivided into 4 biozones based on its foraminiferal content.

Zone AP3 (190-140 m) contains boreal and boreal-lusitanian faunas indicating a water depth of at least $60-100 \mathrm{~m}$. This zone is referred to the Eemian Interglacial. The Late Saalian/Eemian transition is identified at $190.2 \mathrm{~m}$ depth (AP4). The faunas of zone AP2 (140-113 m) record the onset of a major deterioration in climate and a considerable shallowing of the marine environment. The climate is then seen to fluctuate between arctic and boreal-arctic in zone AP1 (113 m to at least $86 \mathrm{~m}$ ). This indicates the succeeding stadial and interstadial periods. Zones AP2 and AP1 are referred to the Early and Middle Weichselian.

The foraminiferal zones at Apholm are compared with the marine sequence at Skærumhede. In addition, an attempt is made to relate these zones to the oxygen isotope chronology and to the Late Quaternary climatic regimes of the Norwegian Sea.
\end{abstract}

K. L. Knudsen, Department of Micropaleontology, Institute of Geology, University of Aarhus, 8000 Arhus C, Denmark. March 28th, 1984.

The Quaternary stratigraphy of the Frederikshavn area was originally described by Jessen et al. (1910). They subdivided the marine sequence at Skærumhede using molluscs. More recently their material has been re-examined as more borehole material became available. A detailed examination of the foraminiferal assemblages, found in the boreholes at Skærumhede (fig. 1), has resulted in a well defined series of biozones being established in the succession (Konradi and Knudsen, 1974; Knudsen, 1976; Knudsen and Lykke-Andersen, 1982). One major drawback of these studies has however, been the lack of available undisturbed core material, and the risk of contamination could not therefore be easily overlooked.

This problem has now been alleviated by the Danish Geological Survey, who made available undisturbed core samples to the author. These came from a borehole put down in 1941 at Apholm, north of Frederikshavn (fig. 2). The borehole had a ground elevation of $3.5 \mathrm{~m}$ above present-day sea-level and penetrated $202.6 \mathrm{~m}$ of Quaternary deposits. The interval between 86.3 $\mathrm{m}$ and $190.2 \mathrm{~m}$ was cored, and the foraminiferal content of 57 core samples was examined from this interval. As samples of this quality have not previously been studied from the present area, the results presented here establish a more satisfactory biostratigraphy, which may therefore also

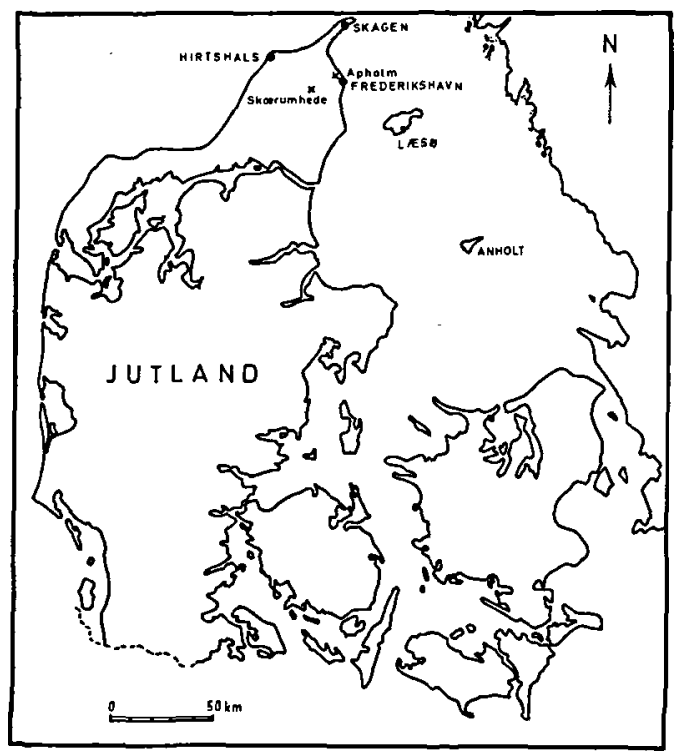

Fig. 1. Locality map. 


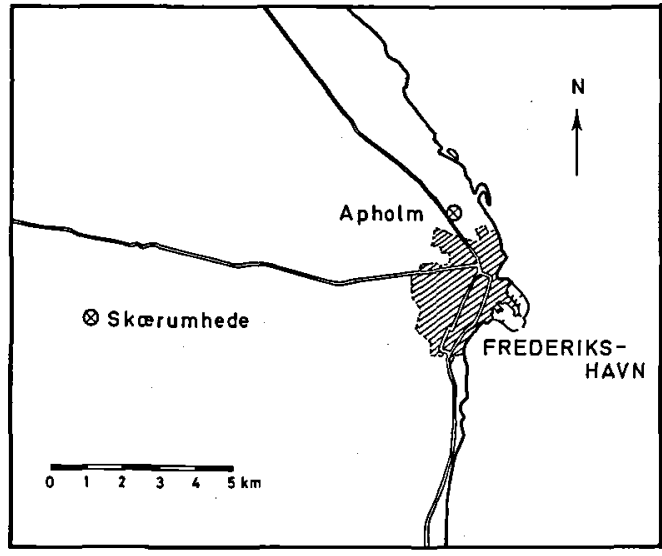

Fig. 2. The location of the Apholm boring 7.131b and of the Skærumhede borings.

act as a re-evaluation of the earlier investigations from Skærumhede.

In addition to the cored material at Apholm, three further samples from deeper levels in the same borehole (190.2-202.6 m) were available for analysis. These had already been washed, and only sediment greater than $0.1 \mathrm{~mm}$ remained. They have been included in the present study in order to complete the succession.

\section{Methods}

The samples from Apholm were treated for foraminiferal analyses according to the laboratory methods described by Feyling-Hanssen et al. (1971) and Meldgaard and Knudsen (1979). At least 300 specimens were counted where possible for quantitative purposes. The percentage frequencies of selected species are illustrated by symbols on the range chart, fig. 3a. In samples with few foraminifera the entire content was analysed. The actual number of tests for each species has been entered on the range chart, where this did not total 100 specimens.

The faunal diversity and dominance indices used on fig. 3b, were defined by Walton (1964). Faunal diversity is the number of ranked species that accounts for $95 \%$ of a counted fauna, and the faunal dominance is the percentage of the most common species in a fauna. These measures, together with the other parameters shown on fig. $3 b$, give an indication of the marine-ecolo- gical conditions during deposition. The species, which were included in calculating boreal content of the faunas, are listed below. The actual percentage values of the species on fig. $3 \mathrm{a}$ are given on the diagram, fig. $3 \mathrm{c}$. An outline of the lithology and the grain size distribution in Apholm $7.131 \mathrm{~b}$ is shown on the left sides of figs. $3 \mathrm{a}$ and $3 \mathrm{~b}$.

The foraminiferal "zones" in the present work are assemblage zones, according to the definition given by Hedberg (1976), but the term "zone" is used for the purpose of brevity.

\section{Foraminiferal zonation and palaeoecology}

The marine sequence of Apholm boring 7.131b is subdivided into four foraminiferal biozones, viz. zones AP4, AP3, AP2 and AP1. The three samples mentioned above from the bottom of the borehole (200-202 $\mathrm{m}$ depth) contained very few foraminifera (figs. $3 a$ and $3 b$ ). One of the samples included a large number of bryozoan fragments. These lowermost samples are considered here to represent a non-marine Quaternary deposit.

The foraminiferal zone AP4 is represented by only one sample of stiff clay from 190.15-190.25 $m$ depth. The fauna is in fact a mixture of an arctic and a boreal fauna, but with arctic species as the dominant component (fig. 3a). The most common arctic species are Islandiella helenae, Stainforthia loeblichi, Cassidulina reniforme, Elphidium excavatum and Islandiella norcrossi. A common boreal species is Cassidulina laevigata. The admixing of these two faunas may have resulted from a transition from colder to more temperate conditions during deposition. Presumably the assemblage does not represent an indigenous living fauna. Each sample covers a certain time interval, this one may cover both the final arctic and the beginning of a warmer period. Bioturbation is also a factor, which may result in a considerable mixing of sediments, but even so the arctic components in zone AP4 show that a marine environment had already been introduced into the area, before warmer conditions were fully established.

The clay sequence from 190 to $140 \mathrm{~m}$ depth is included in the foraminiferal zone AP3. Bulimina 
marginata and Cassidulina laevigata are dominant species, and normally Hyalinea balthica is frequent too. Nonion barleeanum, Cassidulina $a b$ tusa and Epistominella exigua are especially common in the lower part of the zone. Pullenia osloensis, Gyroidina umbonatus, Uvigerina peregrina and Globobulimina turgida also become important faunal elements within certain intervals of zone AP3 (figs. 3a and 3c).

The most common miliolid species in zone AP3 are Quinqueloculina seminulum, Miliolinella subrotunda, Pyrgo williamsoni, Sigmoilopsis schlumbergeri, Sigmoilopsis tenuis and Quinqueloculina padana. The total content of miliolids in each of the samples is shown on fig. 3b. It is initially low, but levels off at between 5 and $10 \%$ of the fauna in most of the zone, peaking at $147 \mathrm{~m}(25 \%)$.

The faunal diversity index usually varies from 10 to 20 in most of the samples from zone AP3, and the number of benthonic species is normally between 35 and 45 . Planktonic foraminifera are largely restricted to a few samples between $167 \mathrm{~m}$ and $147 \mathrm{~m}$, rising to $7.5 \%$ of the total counted assemblage at $165.5 \mathrm{~m}$.

In general the present zone AP3 faunas reflect boreal to boreal-lusitanian marine-ecological conditions, water depths of at least $60-100 \mathrm{~m}$ and normal marine salinities of at least $33 \%$ (Höglund, 1947; Lange, 1956; Jarke, 1961; Risdal, 1964; Gevirtz et al., 1971; Thiede et al., 1981; van Weering and Qvale, 1983). The presence of planktonic foraminifera in a few samples may be an indication of more open connection to the Norwegian Sea at these levels in the borehole.

In the upper part of zone AP3 there are some drastic changes in dominance. In one sample (no. 30) Stainforthia fusiformis is the most frequent species instead of the usual dominance of Bulimina marginata or Cassidulina laevigata. In the overlying sample Bolivina spathulata reaches a peak, and the fauna is subsequently dominated by miliolids. The gaps between these samples are unfortunately too large $(5 \mathrm{~m})$ to give any detailed information about the environmental significance of these variations. All the important species here belong in water depths of up to about $100 \mathrm{~m}$, while a relatively large number of miliolids could indicate increased salinities (Murray, 1973). More closely spaced sampling from the upper part of zone AP3 might lead to a better under- standing of the apparently changing conditions reflected in the foraminifera towards the end of that warm period.

The lusitanian species Quinqueloculina padana, which is common in recent Mediterranean faunas (Giunta, 1954) but not found in the Recent Skagerrak (see also Knudsen and LykkeAndersen, 1982), occurs throughout most of zone AP3 (figs. 3a and 3c). The faunas indicate a period of true interglacial conditions, when the environment must have been warmer than in the present Kattegatt and Skagerrak.

Environmental conditions are totally altered in zone AP2 (140-113 m depth). The sediment is still largely a clay, but with a gradual increase in sand-sized material upwards. The deep, warmwater indicator-species such as Bulimina marginata, Cassidulina laevigata, Hyalinea balthica and many others disappear in this zone and are replacèd by a shallower water assemblage, viz. $E l$ phidium excavatum, E. margaritaceum, E. gerthi, $E$. albiumbilicatum and Nonion depressulum. Both Elphidium gerthi and $E$. excavatum began to appear in the upper part of zone AP3, a possible indication that a slight shallowing of the water had already commenced, but a significant drop in sea-level must have occurred at the transition between zone AP3 and zone AP2. At the beginning of zone AP2 the water depth was probably less than $20 \mathrm{~m}$, but there seems to have been a return to slightly deeper water again in the upper part of the zone. The shallow water indicatorspecies become relatively less frequent, and some of the deeper water species known from zone AP3 return in low frequencies to the faunas.

Indicators of cooler marine conditions are first introduced at the bottom of zone AP2 and increase in importance upwards through the zone. An increase of the arctic Cassidulina reniforme also supports the suggestion of deeper water in the upper part of zone AP2, because $C$. reniforme prefers normal marine salinities, and it only occurs in large numbers at water depths of more than about $20 \mathrm{~m}$ (Buzas, 1965; Nagy, 1965).

Both the number of species and the number of benthonic specimens are extremely high in the upper part of this zone. The reduction in specimen numbers during the shallower period at the base of the zone may be an indication of a higher rate of sedimantation. No planktonic specimens are found in zone AP2, and the content of mili- 


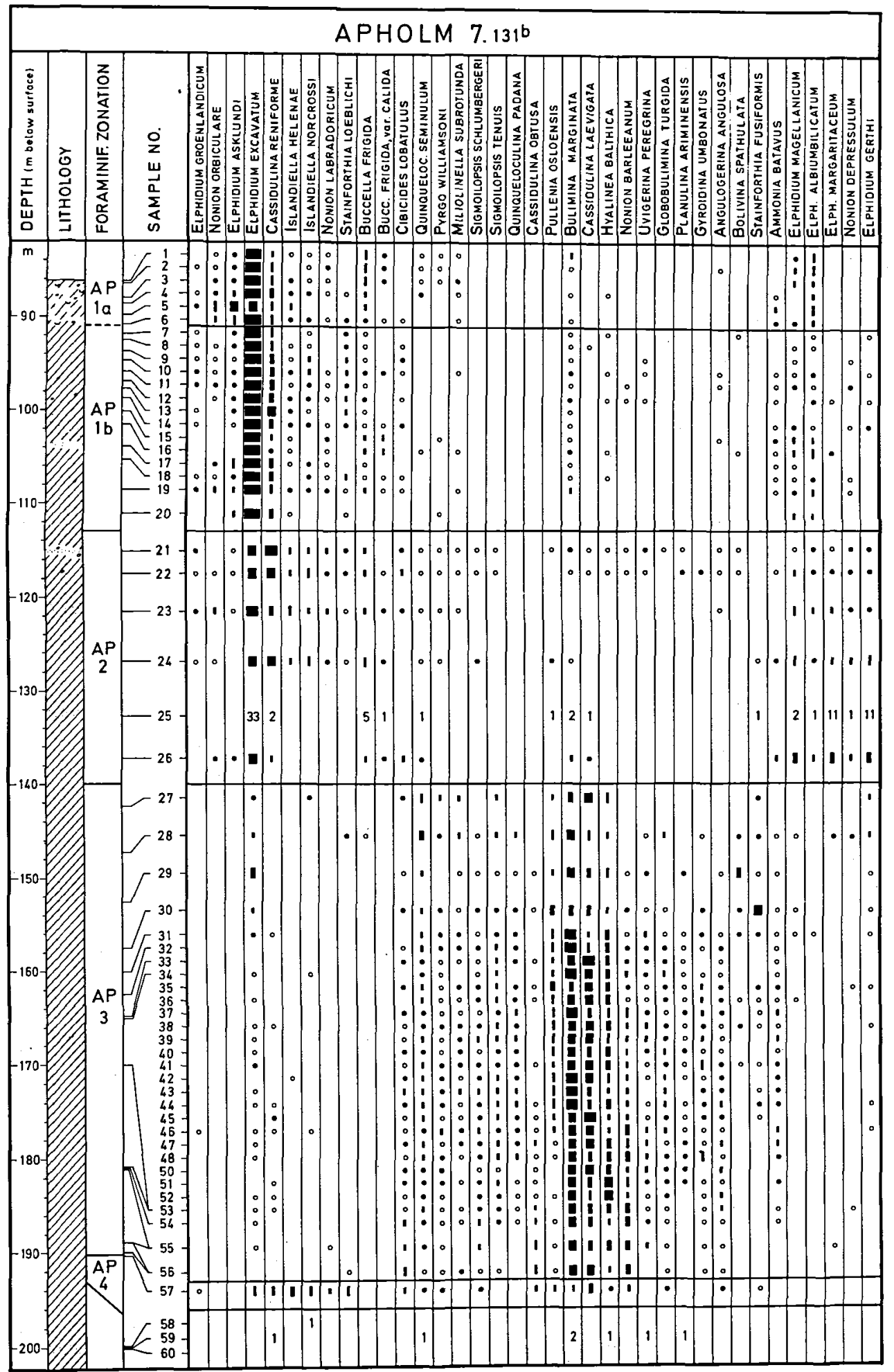

Fig. 3a. Range chart for Apholm boring 7.131b with frequency distribution of selected foraminiferal taxa. Legend on fig. 4. The samples from 35 to 52 are all from the interval between 165 and $170 \mathrm{~m}$. 


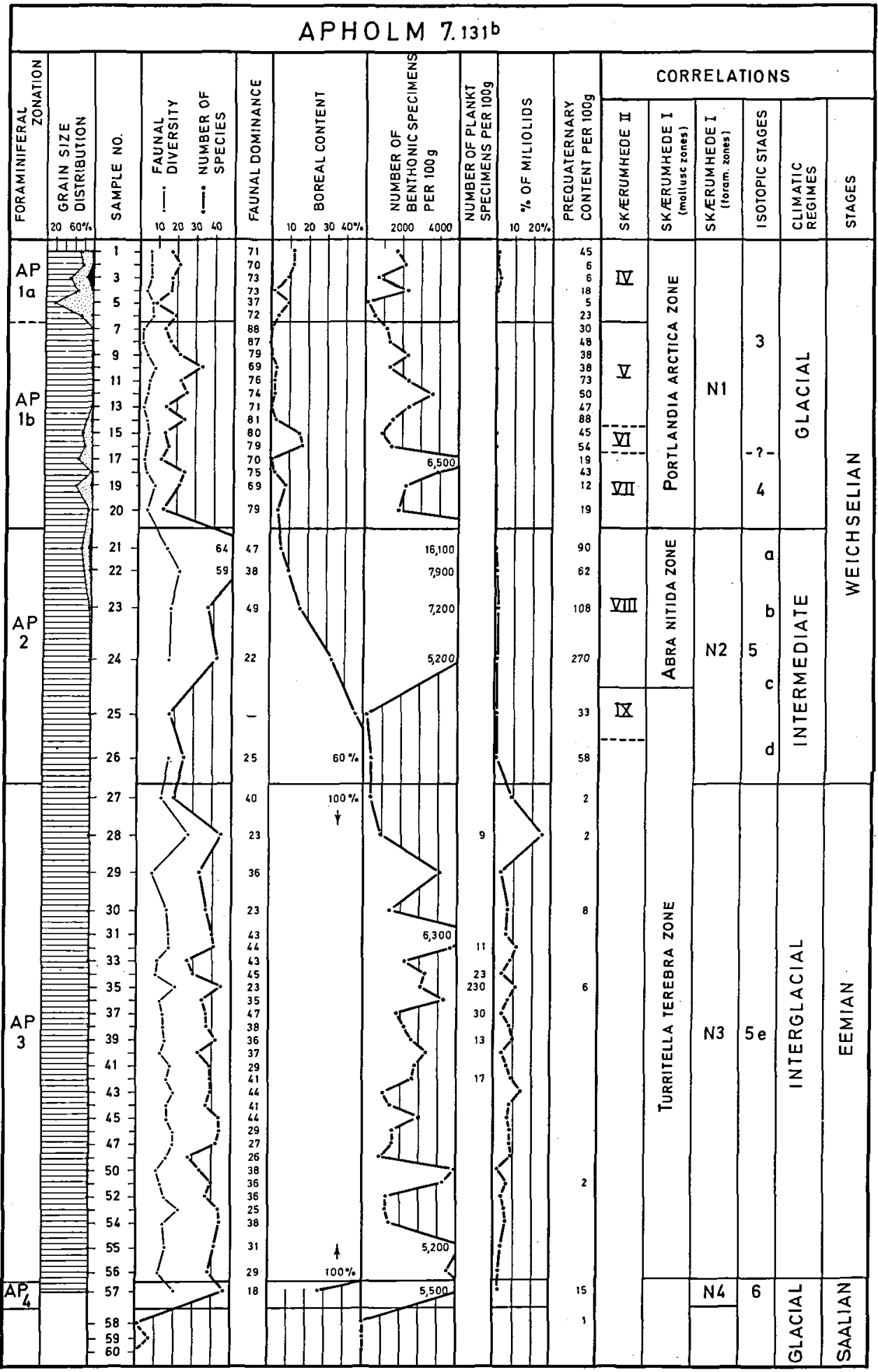

Fig. 3b. Apholm boring 7.131b, palaeoenvironmental indices and correlations. Isotope stages are those established by Shackleton and Opdyke (1973), and climatic regimes are according to Belanger (1982). Legend for grainsize distribution on fig. 4. 
A P HOLM $7.1311^{\circ}$

\begin{tabular}{|c|c|c|c|c|c|c|c|c|c|c|c|c|c|c|c|c|c|c|c|c|c|c|c|c|c|c|c|c|c|c|c|c|c|c|c|c|c|}
\hline 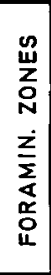 & 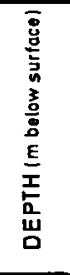 & $\begin{array}{l}0 \\
2 \\
u \\
\overrightarrow{1} \\
\frac{0}{\Sigma} \\
\vdots \\
0 \\
0\end{array}$ & 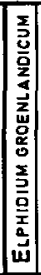 & 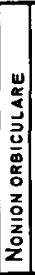 & 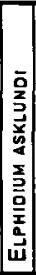 & 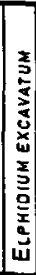 & 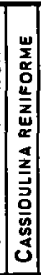 & 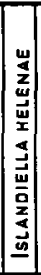 & 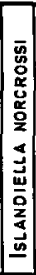 & 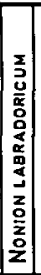 & 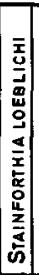 & 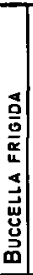 & 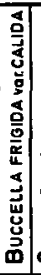 & & 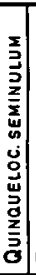 & 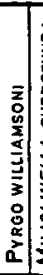 & 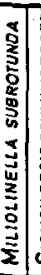 & 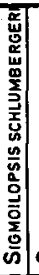 & 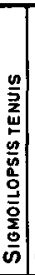 & 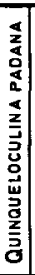 & 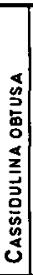 & 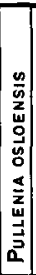 & 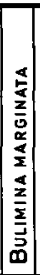 & 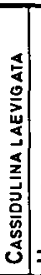 & 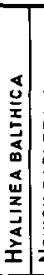 & 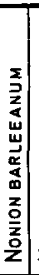 & 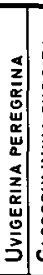 & 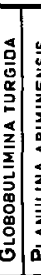 & 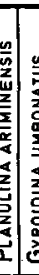 & & $\mid$ & 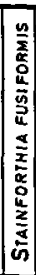 & 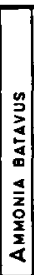 & & 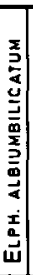 & & 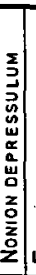 \\
\hline & & & & & & & & & & & & & & & & & & & & & & & & & & & & & & & & & & & & & \\
\hline & 86.3 & $\mathbf{1}$ & & - & 1 & 71 & 5 & + & + & 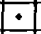 & & 7 & 1 & & $\div$ & $\cdot 1$ & 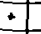 & & & & & & 2 & & & & & & & & & & & & 9 & & \\
\hline $\mathbf{P}$ & 86.6 & 2 & $\circ$ & $\cdot$ & 1 & 70 & 6 & & $\because$ & 1 & & 7 & 1 & & $\dot{\circ}$ & 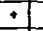 & & & & & & & 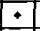 & & & & & & & $\div$ & & & & 2 & 8 & & \\
\hline & 88.0 & 3 & & \begin{tabular}{|l|}
1 \\
\end{tabular} & \begin{tabular}{|l|}
1 \\
\end{tabular} & 73 & 6 & 1 & + & + & & 8 & 1 & & $\cdot$ & $\cdot 1$ & 1 & & & & & & - & & & & & & & & & & & 3 & 5 & & \\
\hline & 88.6 & 4 & \begin{tabular}{|l|}
+ \\
\end{tabular} & \begin{tabular}{|l|}
1 \\
\end{tabular} & 5 & 73 & 15 & 1 & 1 & $\cdot 1$ & + & 3 & & & 1 & & $\therefore$ & & & & & & + & & + & & & & & & & & + & & 2 & & \\
\hline & 89.8 & 5 & 1 & 9 & 37 & 31 & 6 & 5 & & & & 2 & & & & & & & & & & & & & & & & & & & & & 4 & & 6 & & \\
\hline & 90.8 & 6 & & 2 & 9 & 72 & 9 & 1 & 1 & 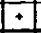 & 1 & 1 & $\cdot$ & $\cdot$ & & & $\div$ & & & & & & + & & & & & & & & 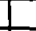 & & 1 & 1 & 2 & & \\
\hline & 91.8 & 7 & $\cdot$ & & 1 & 88 & 8 & & $\because$ & & 1 & + & & - & & & & & & & & & $\because$ & & $\bullet$ & & & & & & + & & 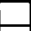 & $\square$ & $\cdot$ & & \\
\hline & 92.5 & 8 & +1 & \begin{tabular}{|l|}
+ \\
\end{tabular} & 1 & 87 & 8 & + & $\div$ & & 1 & 4 & & + & & & & & & & & & +1 & + & & & & & & & & & & + & + & & \\
\hline & $93.8^{\circ}$ & 9 & + & + & + & 79 & 13 & $\cdot$ & 2 & & 3 & + & & 1 & & & & & & & & & + & & & & + & & & & & & 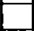 & + & - & & + \\
\hline & 94.6 & 10 & 1 & +4 & 1 & 70 & 18 & 1 & 2 & + & 3 & 1 & 1 & + & & & $\cdot$ & & & & & & 1 & & + & & 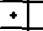 & & & + & & & $\cdot$ & + & 1 & & \\
\hline & 95.9 & 11 & 1 & 1 & 1 & 76 & 16 & 7 & 1 & +1 & 1 &. & & & & & & & & & & & + & & & + & & & & $\div$ & & &. & 1 & 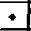 & & 1 \\
\hline & 97.0 & 12 & & + & 1 & 74 & 16 & 1 & 1 & 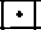 & 3 & 1 & & \pm & & & & & & & & & 1 & & + & + & \pm & & & & & & + & & 1 & $\div$ & \\
\hline & 97.7 & 13 & 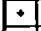 & & 1 & 71 & 24 & 1 & \pm & & 2 & $\cdot$ & & & & & & & & & & & $\cdot$ & & & & & & & 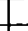 & & & 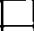 & - & - & & $\ldots$ \\
\hline & 98.8 & 14 & +1 & & 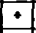 & 81 & 13 & 1 & 1 & $\cdot$ & 1 & + & +1 & 1 & & & & & & & & & + & & & & & & & & & & 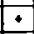 & 1 & $\cdot$ & & $\cdot$ \\
\hline & 100.2 & 15 & & & & 80 & 5 & $\div$ & & 1 & & 2 & 3 & & & + & & & & & & & + & & & & & & &. & & & 1 & \begin{tabular}{|l|}
3 \\
\end{tabular} & 4 & & \\
\hline & 101.3 & 16 & & & & 79 & 1 & $\div$ & & + & & 2 & 5 & & +1 & & + & & & & & & 1 & & \begin{tabular}{|l|}
+ \\
\end{tabular} & & & & & 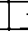 & 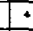 & & + & 3 & 7 & 1 & \\
\hline & 1040 & 17 & & 1 & 6 & 70 & 19 & + & 1 & 1 & & $\cdot$ & & & & & & & & & & & 1. & & & & & & & 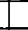 & & & 4 & + & & & \\
\hline & 105.4 & 18 & 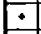 & + & 1 & 75 & 18 & & 1 & . & 2 & + & + &. & & & & & & & & & $\cdot$ & & + & & - & & & & & & + & $\div$ & 1 & & $\div$ \\
\hline & 108.6 & 19 & \begin{tabular}{|l|}
1 \\
\end{tabular} & \begin{tabular}{|l|}
2 \\
\end{tabular} & 4 & 69 & 10 & 1 & 1 & 11 & \begin{tabular}{|l|}
1 \\
\end{tabular} & 3 & + & + & & & 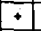 & & & & & & 2 & & & & & & & & & & . & 1 & 5 & & $\cdot$ \\
\hline & 111.8 & 20 & & & 2 & 79 & 13 & 1 & & & 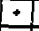 & & & & & - & & & & & & & & & & & & & & 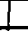 & & & & 2 & 2 & & \\
\hline & 115.1 & 21 & 1 & & + & 28 & 47 & 3 & 4 & \begin{tabular}{|l|}
2 \\
\end{tabular} & \begin{tabular}{|l|}
1 \\
\end{tabular} & 2 & & 1 & + & + & \pm & + & + & & & + & 1 & + & 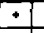 & + & 1 & + & 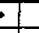 & + & \pm & & 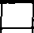 & + & 1 & & 1 \\
\hline & 117.5 & 22 & \begin{tabular}{|l|}
$\div$ \\
\end{tabular} & + & + & 26 & 38 & 5 & 9 & 1 & 1 & 2 & + & 2 & \pm & $\cdot$ & 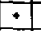 & 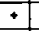 & + & & & 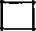 & $\cdot 1$ & + & $\bullet$ & $*$ & $\cdot$ & & 11 & + & + & & 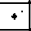 & 3 & 1 & 1 & 1 \\
\hline AP & 121.4 & 23 & 1 & \begin{tabular}{|l|}
2 \\
\end{tabular} & $\div$ & 69 & 13 & 6 & 4 & 2 & + & 3 & 1 & 1 & + & $\circ$ & + & & & & & & & & & & - & & & + & & & 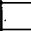 & 6 & 3 & 2 & 1 \\
\hline & 126.9 & 24 & \begin{tabular}{|l}
+ \\
\end{tabular} & \begin{tabular}{|l|}
+ \\
\end{tabular} & & 22 & 21 & 5 & 7 & 1 & + & 7 & $\sqrt{1}$ & & + & $\cdot$ & & 1 & & & & 1 & $\because$ & & & & & & & & & + & \begin{tabular}{|l|}
1 \\
\end{tabular} & 10 & 1 & 5 & \begin{tabular}{l|l} 
\\
\end{tabular} \\
\hline & 132.8 & 25 & & & & 3 & 2 & & & & & 5 & 1 & & 1 & & & & & & & 1 & 2 & 1 & & & & & & & & 17 & & 2 & 8 & 11 & 1 \\
\hline & 137.4 & 26 & & \begin{tabular}{|l|}
1 \\
\end{tabular} & & 25 & 5 & & & & & 5 & 1 & $\begin{array}{ll}3 \\
\end{array}$ & 1 & & & & & & & & 3 & 1 & & & & & & & 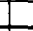 & & 3 & 12 & 3 & II & 5 \\
\hline & 142.8 & 27 & & & & 1 & & & 1 & & & & & 1 & 8 & 2 & 3 & & 3 & & & 2 & 20 & 40 & 8 & & & & & & & $T$ & & $\square$ & & & \\
\hline & 147.3 & 28 & & & & 3 & & & & & 11 & $\div$ & & & 15 & 1 & 2 & +1 & 4 & 3 & & 6 & 23 & \begin{tabular}{|l|}
10 \\
\end{tabular} & 4 & & + & 2 & + & & 1 & 1 & $\cdot$ & + & & 1 & 1 \\
\hline & 152.6 & 29 & & & & 14 & & & & & & & & $\cdot$ & 2 & & + & +1 & +1 & + & + & 6 & 36 & 14 & 7 & + & 1 & & 1 &. & 17 & + &. & - & & & \\
\hline & 157.6 & 30 & & & & 5 & & & & & & & & 1 & 3 & 1 & $\cdot$ & 1 & 1 & 1 & + & 11 & 17 & 17 & 5 & 1 & + & + & 1 & & 1 & 23 & $\dot{-}$ & + & & & \\
\hline & 159.9 & 31 & & & & 1 & $\cdot$ & & & & & & & & 2 & 1 & 1 & + & 1 & 1 & & 10 & 43 & \begin{tabular}{|l|l|}
5 \\
\end{tabular} & 20 & + & 4 & + & $\cdot 1$ & + & 1 & 1 & 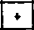 & + & + & & \\
\hline & 162.5 & 32 & & & & & & & & & & & & + & 4 & 1 & 1 & 1 & 3 & 1 & & 9 & 44 & 10 & 13 & \begin{tabular}{|l|}
1 \\
\end{tabular} & 1 & 1 & + & 1 & & & $\cdot$ & & & & \\
\hline & 164.9 & 33 & & & & & & & & & & & & 1 & 1 & $\div$ & + & $\cdot$ & 5 & 1 & + & 4 & 25 & \begin{tabular}{|l|}
43 \\
\end{tabular} & 13 & 1 & 1 & & - & 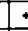 & & &. & & & & \\
\hline & 165.3 & 34 & & & & + & & & $\therefore$ & & & & & & 1 & $\div$ & & +1 & 3 & & & \begin{tabular}{|l|}
3 \\
\end{tabular} & 45 & 26 & 12 & \begin{tabular}{|l|}
3 \\
\end{tabular} & 1 & 2 & +1 & 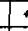 & & & + & & & & \\
\hline & 165.5 & 35 & & & & & & & & & & & & 1 & 3 & + & 1 & 1 & 4 & 1 & + & 11 & 19 & 23 & \begin{tabular}{|l|}
19 \\
\end{tabular} & 1 & $\dot{\square}$ & 1 & +2 & 1 & & 1 & 1 & & & & + \\
\hline & 165.7 & 36 & & & & $\cdot$ & & & & & & & & & 3 & 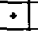 & + & 1 & 2 & 1 & + & 6 & 27 & 35 & 15 & +1 & $\div$ & $\overline{1}$ & 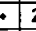 & 1 & $\therefore$ & $\cdot$ & + & + & & & \\
\hline & 166.0 & 37 & & & & & & & & & & & & 1 & 1 & $\div$ & +4 & $\cdot 1$ & 1 & 2 & & 9 & 47 & 12 & 7 & \begin{tabular}{|l|}
7 \\
\end{tabular} & 1 & + & it & 1 & & + & $\sqrt{2}$ & & & & \\
\hline & 166.1 & 38 & & & & 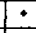 & + & & & & & & & $\cdot$ & 1 & 1 & 1 & 1 & 3 & 1 & & 7 & 38 & 25 & \begin{tabular}{|l|}
11 \\
\end{tabular} & \begin{tabular}{|l|}
3 \\
\end{tabular} & 2 & $i$ & + & & 1 & 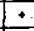 & + & & & & \\
\hline & 166.3 & 39 & & & & $\cdot$ & & & & & & & & $\cdot$ & 3 & $\because$ & 1 & 2 & 3 & 2 & & 6 & 36 & \begin{tabular}{|l|}
21 \\
\end{tabular} & \begin{tabular}{|c|}
10 \\
\end{tabular} & \begin{tabular}{|l|l|} 
\\
\end{tabular} & 2 & 1 & 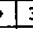 & . & & & 2 & & & & \\
\hline & 166.9 & 40 & & & & + & & & & & & & & 1 & 1 & 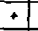 & 1 & .1 & 1 & 1 & & 8 & 37 & 18 & \begin{tabular}{|l|}
19 \\
\end{tabular} & \begin{tabular}{|l|}
4 \\
\end{tabular} & 1 & 3 & 1 & 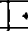 & & & 1. & & & & \\
\hline AP & 167.2 & 41 & & & & 1 & & & & & & & & +1 & 2 & 1 & 1 & 1 & 4 & 1 & + & 5 & 29 & \begin{tabular}{|l|}
22 \\
\end{tabular} & \begin{tabular}{|l|}
17 \\
\end{tabular} & 3 & $\div$ & 1 & 19 & & - & \pm & 1 & & & & \\
\hline 3 & 167.5 & 42 & & & & & & + & & & & & & 1 & 3 & 1 & 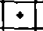 & 1 & 2 & 1 & & 6 & 61 & \begin{tabular}{|l|}
23 \\
\end{tabular} & 9 & 2 & $\div$ & 3 & 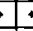 & 1 & & & 1 & & & & \\
\hline & 167.9 & 43 & & & & + & & & & & & & & 2 & 4 & 1 & 2 & 2 & 2 & 2 & & 8 & 44 & 13 & 4 & 2 & & 4 & 1 & 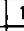 & & + & t & & & & \\
\hline & 168.2 & 44 & & & & + & + & & & & & & & 1 & 2 & \begin{tabular}{|l|l|}
1 \\
\end{tabular} & +1 & 1 & 2 & 2 & + & 8 & 41 & 16 & 14 & 2 & + & 2 & +1 & 2 & & 1 & 11 & & & & \\
\hline & 168.5 & 45 & & & & & 1 & & & & & & & $\cdot$ & 3 & + & $\cdot 1$ & 1 & 2 & 1 & $\cdot$ & 6 & 29 & 44 & 3 & 4 & & & $\cdot 1$ & 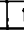 & & + & & & & & \\
\hline & 158.9 & 46 & 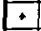 & & & $\div$ & $1+$ & & + & & & & & + & 1 & 2 & 1 & 2 & 1 & 1 & + & 3 & 26 & \begin{tabular}{|l|}
29 \\
\end{tabular} & 5 & 12 & 4 & 2 & + & . & & & 3 & & & & \\
\hline & 169.2 & 47 & & & & $\div$ & & & & & & & & 1 & 2 & $\cdot 1$ & 1 & 1 & 3 & 1 & 2 & + & 27 & 21 & 15 & 8 & 5 & + & 11 & 2 & & & 2 & & & & \\
\hline & 169.5 & 4 & & & & + & & & & & & & & $\cdot$ & 3 & & + & 1 & 2 & 2 & $\div$ & $\cdot$ & 26 & 16 & 18 & 12 & 3 & 1 & 15 & & & & 1 & & & & \\
\hline & 170.1 & 50 & & & & & & & & & & & & 1 & 1 & \begin{tabular}{l|}
1 \\
\end{tabular} & & + & + & & 4 & & 31 & \begin{tabular}{|l|}
38 \\
\end{tabular} & 15 & 4 & 2 & 1 & 1 & 2 & & & & & & & \\
\hline & 170.5 & 51 & & & & & $\div$ & & & & & & & 1 & 1 & \begin{tabular}{|l|}
1 \\
\end{tabular} & $\cdot$ & 1 & 3 &. & $\cdot$ & 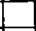 & 36 & \begin{tabular}{|l|}
16 \\
\end{tabular} & 26 & 7 & 1 & 1 & 1 & & & & 1 & & & & \\
\hline & 171.0 & 52 & & & & + & + & & & & & & & 4 & 1 & + & & 1 & 1 & - & $\div$ & \pm & 24 & 9 & 36 & 4 & $\div$ & 1 & . & & & & & & & & \\
\hline & 175.0 & 53 & & & & + & + & & & & & & & + & 2 & + & $\cdot 1$ & 1 & 1 & + & 2 & & 25 & \begin{tabular}{|l|}
17 \\
\end{tabular} & 18 & 12 & + & 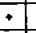 & . & 3 & & & + & & & & $\cdot 1$ \\
\hline & 1810 & 54 & & & & + & & & & & & & & 3 & 1 & $\cdot 1$ & $\cdot 1$ & 3 & 1 & + & 2 & + & 38 & 15 & 5 & 19 & 1 & + & 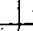 & 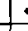 & & & + & & & & \\
\hline & 185.0 & 55 & & & & 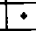 & & & & 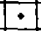 & & & & 2 & 1 & $\cdot$ & & 2 & & & 7 & $\cdot$ & 31 & 12 & 16 & 12 & 5 & • & & & & & & & & & \\
\hline & 189.5 & 56 & & & & & & & & & + & & & 10 & +1 & \begin{tabular}{|l|}
+1 \\
\end{tabular} & 1 & + & + & & 9 & + & 29 & 25 & 6 & 11. & & + & & & & & & & & & \\
\hline $\overrightarrow{A R}$ & 1902 & 57 & + & & & 7 & 10 & 17 & 6 & 5 & 8 & & & 5 & 1 & 1 & & 1 & & & 2 & 4 & 2 & 18 & 1 & 5 & & 1 & & 1 & & $\dot{\dot{ }}$ & & & & & \\
\hline & 200.0 & 58 & & & & & & & 1 & & & & & & & & & & & & & & & & L & & & & & & & & & & & & \\
\hline & 201.7 & 5 & & & & & 1 & & & & & & & & 1 & & & & & & & & 2 & & 1 & & & & & & & & & & & & \\
\hline & 202.2 & 60 & & & & & & & & & & & & & & & & & & & & & & & & & & & & & & & & & & & \\
\hline
\end{tabular}

Fig. 3c. Percentage values of the 37 selected species on fig. 3a. Values less than $0.5 \%$ are marked with a cross. Numbers in italic indicate the actual number of specimens, where counts were too low for quantitative analysis. The depth is given as an average of the sample thickness. 


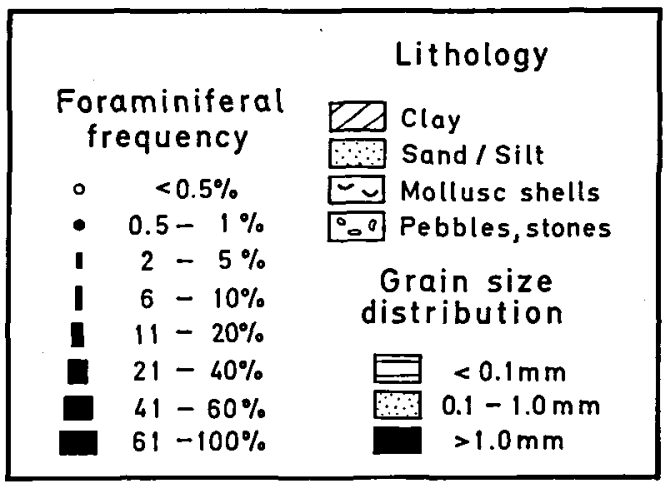

Fig. 4. Legend for figs. 3a and 3b.

olids is extremely low. The lower water depth combined with reduced salinities presumably excluded those two groups from the faunas.

The faunas of zone AP2 are boreal to borealarctic in character, and this zone is therefore interpreted as a transition period between the preceding warm and a subsequent mainly arctic period.

The sequence between $113 \mathrm{~m}$ depth and the uppermost sample at $86.3 \mathrm{~m}$ is included in foraminiferal zone AP1. The sediment varies much more in this part of the marine sequence. The lower part of zone AP1 is mainly clay with a content of sand and a few pebbles. It is overlain by $9 \mathrm{~m}$ of clay between 100 and $91 \mathrm{~m}$. The sediment finally coarsens, consisting of alternating layers of clayey sand, coarse sand and sandy clay with scattered pebbles and fragments of mollusc shells and plant material.

Zone AP1 has been subdivided into two subzones (AP1a and AP1b). In general, the faunas in zone AP1 are characteristically dominated by Elphidium excavatum and Cassidulina reniforme. At certain levels Elphidium asklundi is quite common, and other important accessory species in zone AP1 include Nonion orbiculare, Islandiella helenae, I. norcrossi, Stainforthia loeblichi, Buccella frigida, Elphidium magellanicum and $E$. albiumbilicatum (fig. 3a). The faunas are mainly arctic except at two intervals, where the boreal content increases to significant levels (fig. 3b), viz. at about 102-100 m (samples 16 and 15) and again from $91 \mathrm{~m}$ depth to the uppermost sample at $86.3 \mathrm{~m}$ (subzone AP1a). These alternating faunal elements suggest the presence of two stadials and two interstadials in zone AP1.
The uppermost part of the marine sequence was not cored at Apholm 7.131b. Some cutting samples were however, available from an adjacent borehole (Apholm 7.131a). This material was unfortunately difficult to interpret. A sample from $80 \mathrm{~m}$ depth for example, possessed an arctic foraminiferal fauna. A barren zone occurred around $65 \mathrm{~m}$, but arctic faunas returned in the overlying sediments. The upper boundary of the marine sequence could not be defined on this material. To do so would require a more detailed biostratigraphical study of these sediments making use of undisturbed core material.

\section{Correlations and age}

The foraminiferal zones in Apholm 7.131b can be correlated with corresponding zones described from Skærumhede I (Knudsen and Lykke-Andersen, 1982) and from Skærumhede II (Konradi and Knudsen, 1974; Knudsen, 1976). An attempt has also been made on fig. $3 \mathrm{~b}$ to correlate these zones with oxygen isotope stages established by Shackleton and Opdyke (1973).

The arctic zone AP4 in Apholm corresponds to zone N4 in the Skærumhede I boring (Knudsen and Lykke-Andersen, 1982). The faunas in these two zones are very similar. Accessory species such as Islandiella helenae and Stainforthia loeblichi are common in both. These arctic zones are considered to represent the Late Saalian corresponding to oxygen isotope stage 6 (Shackleton and Opdyke, 1973). An age of about 127,000 BP has been mentioned by e.g. Kellogg et al. (1978), Ruddiman et al. (1980), Belanger (1982) and Streeter et al. (1982) for the boundary between oxygen isotope stages 6 and 5 .

Zone AP3 with its boreal to boreal-lusitanian deep water faunas is correlated with zone N3 of the Skærumhede I boring. The faunal composition is similar, and even details in the faunal succession through the zone can be compared. Nonion barleeanum occurs with higher frequencies in the lower part of each zone, miliolid species are important in the upper part, and a slight increase in shallow water species, such as Elphidium excavatum and $E$. gerthi occurs in the uppermost samples of both zones AP3 and N3. Only few faunas from zone N3 of the Skærumhede I boring have however, been analysed clo- 
sely (Knudsen and Lykke-Andersen, 1982), and some details are therefore missing from that boring. Planktonic specimens found in zone AP3 at Apholm, have for instance not been found in Skærumhede I.

Zones AP3 and N3, which reflect true interglacial conditions, are here referred to the Eemian Interglacial, and a correlation with oxygen isotope stage 5e (Shackleton and Opdyke, 1973) is suggested. The upper boundary of stage $5 \mathrm{e}$ is placed at about 115,000 BP by Kellogg et al. (1978), Ruddiman et al. (1980) and Streeter et al. (1982). If this correlation is correct, the $50 \mathrm{~m}$ thick zone AP3 was deposited within at time period of about 12,000 years. A corresponding foraminiferal zone is found in many other borings from the Frederikshavn area. The thickness varies from 25 to $60 \mathrm{~m}$ over the whole area (Knudsen, 1984). Eemian faunas from a section at Fjøsanger in western Norway have been described by Mangerud et al. (1981). Beds $\mathrm{N}$ to $\mathrm{J}$ from that section were correlated with oxygen isotope stage 5e (see also Mangerud et al., 1979). FeylingHanssen (1981) described Eemian Interglacial faunas from a boring in the northern North Sea. His Eemian zone W was later (Feyling-Hanssen, 1982) placed in oxygen isotope stage 5e. In that area the Eemian deposits were only a few metres in thickness.

Recently published Ionium-dates (Vogel, 1982) support an age of about 120,000 years for the peak sea-level during the Eemian (Zagwijn, 1983).

Zone AP2 at Apholm, containing faunas reflecting the transition between the boreal-lusitanian environments of zone AP3 and the mainly arctic zone AP1, corresponds to zone $\mathrm{N} 2$ of the Skærumhede I boring (Knudsen and Lykke-Andersen, 1982). The sample number is low in zone AP2, but despite this, it is apparent that the upper part of that zone, which is rich in Cassidulina reniforme, corresponds to zone VIII at Skærumhede II (Konradi and Knudsen, 1974), whereas the lower part with very few $C$. reniforme can be correlated with zone IX.

The palaeoenvironmental conditions, which existed during the deposition of zone AP2, are compatible with the Early Weichselian intermediate climatic regime described by e.g. Kellogg (1980) and Belanger (1982) from the Norwegian Sea. This period is correlated with oxygen iso- tope stages 5a-d established by Shackleton and Opdyke (1973). They can also be compared with the climatic response patterns (CRP) described by Belanger (1982). The present faunas do not follow the same pattern as the benthonic deep water faunas of the Norwegian Sea (CRP2), which suggest that a rather uniform temperature existed from stage 5e through to stage $5 \mathrm{~d}$. It is not the same as the planktonic evidence (CRP1) either, which betokens an abrupt temperature change between stage $5 \mathrm{e}$ and $5 \mathrm{~d}$. The foraminiferal faunas in zone AP2 at Apholm on the other hand indicate a gradual change to cooler conditions through the zone. As mentioned by Streeter et al. (1982) and by Belanger (1982), low surface salinities have probably affected the planktonic faunas of the Norwegian Sea during the intermediate climatic regime (5a-d). The sea was presumably covered by ice during the winter (Streeter et al., 1982), and summer melting would give a low-salinity near-surface layer. Such reduced salinities in the surface layer would also influence the shallower water masses as represented by the zone AP2 in Apholm. Evidence for a gradual deterioration in climate may presumably be more clearly reflected in these shallower faunas than in any of the Norwegian Sea faunas mentioned by Belanger (1982).

A eustatic fall in sea-level of $32 \mathrm{~m}$ at the Eemian-Weichselian boundary, which commenced in the Late Eemian, was mentioned by Zagwijn (1977 and 1983) for the southern North Sea area. A drop of about the same order of magnitude was suggested by Jelgersma (1979), who found evidence of a sea-level more than $40 \mathrm{~m}$ lower than the present in the Early Weichselian of the German Bight of the North Sea. Kellogg et al. (1978) mentioned a major drop in sea-level of about 50 $\mathrm{m}$ at $115,000 \mathrm{BP}$ followed by a slight recovery at about $105,000 \mathrm{BP}$. This fits fairly well with the evidence found in the Apholm boring. A slight shallowing in water depth towards the end of zone AP3 is followed by a sudden change to much shallower conditions during deposition of the lower part of zone AP2. In the upper part of this zone the faunas reflect a return to somewhat deeper waters. The abrupt change apparent at the zone AP3-AP2 boundary would nevertheless appear to suggest a decrease in water depth of more than $50 \mathrm{~m}$. Local tectonic movements could also however, have played a role here. 
The boundary between isotope stages $5 \mathrm{a}$ and 4 is placed at 72,000 BP by Streeter et al. (1982). Kellogg et al. (1978) and Ruddiman et al. (1980) mention an age of about 75,000 years.

The mainly arctic foraminiferal zone $A P I$ in Apholm is correlated with the lower part of zone $\mathrm{N} 1$ in the Skærumhede I boring (Knudsen and Lykke-Andersen, 1982). A correlation with zones VII, VI, V and IV in the Skærumhede II boring is also suggested (fig. $3 b$ ), based on the evidence of a changing boreal content in the faunas. The boreal-arctic faunas between 102 and $100 \mathrm{~m}$ depth in Apholm seem to correspond to zone VI in Skærumhede II, which is characterized by a slight increase in the relative proportion of boreal specimens. The faunas in the upper part of subzone AP1b, between 100 and $91 \mathrm{~m}$ depth, compare well with the high-arctic zone $\mathrm{V}$ faunas in Skærumhede II. There is a very high dominance of Elphidium excavatum and decreasing percentages of Cassidulina reniforme through that zone. The increased boreal content present in subzone AP1a, together with a coarsening sediment containing gravel and plant remains, is also seen in zone IV of Skærumhede II (Bahnson, et al., 1974) and in zone E/F of the coastal cliff at Hirtshals (Lykke-Andersen, 1971). Radiocarbon dates from zone $E$ in Hirtshals suggest an age corresponding to the Middle Weichselian Moershoofd Interstadial (Lykke-Andersen, 1981 and 1982). Zone AP1 in Apholm thus represents a continuation of the marine succession from the Early Weichselian starting in zone AP2 into the Middle Weichselian. Zone AP1 seems to include oxygen isotope stage 4 and a part of stage 3 (Shackleton and Opdyke, 1973), and it apparently belongs within the glacial climatic regime before Termination I (cf. Ruddiman et al., 1980; Belanger, 1982).

\section{Foraminiferal list}

The following two lists include the foraminiferal species, which accounted for more than $1.0 \%$ of the fauna in at least one of the samples from Apholm 7.131b. In addition to the listed species, the faunas often contained many species with frequencies of less than $1.0 \%$, especially in zones AP2 and AP3. The planktonic species in zone AP3 are not mentioned here.
A. Species used in calculating the boreal content on the diagram, fig. $3 \mathrm{~b}$ :

Ammonia batavus (Hofker, 1951)

Angulogerina angulosa (Williamson, 1858)

Astrononion tumidum Cushman and Edwards, 1937

Bolivina albatrossi Cushman, 1922

Bolivina spathulata (Williamson, 1858)

Buccella frigida (Cushman), var. calida (Cushman and Cole, 1930)

Bulimina marginata d'Orbigny, 1826

Buliminella elegantissima (d'Orbigny, 1839)

Cassidulina laevigata d'Orbigny, 1826

Cassidulina obtusa Williamson, 1848

Cassidulina subglobosa Brady, 1881

Elphidium albiumbilicatum (Weiss, 1954)

Elphidium gerthi van Voorthuysen, 1957

Elphidium incertum (Williamson, 1858)

Elphidium magellanicum Heron-Allen and Earland, 1932

Elphidium margaritaceum Cushman, 1930

Elphidium translucens Natland, 1938

Epistominella exigua Brady, 1884

Epistominella rugosa (Phleger and Parker, 1951)

Gavelinopsis praegeri (Heron-Allen and Earland, 1913)

Globobulimina turgida (Bailey, 1851)

Gyroidina umbonatus (Reuss, 1851)

Hyalinea balthica (Schroeter, 1783)

Nonion barleeanum (Williamson, 1858)

Nonion depressulum (Walker and Jacob, 1798)

Nonion niveum Lafrenz, 1963

Nonionella iridea Heron-Allen and Earland, 1932

Planorbulina distoma Terquem, 1876

Planulina ariminensis d'Orbigny, 1826

Pullenia osloensis Feyling-Hanssen, 1954

Pullenia subcarinata (d'Orbigny, 1839)

Quinqueloculina padana Perconig, 1954

Sigmoilopsis schlumbergeri (Silvestri, 1904)

Sigmoilopsis tenuis (Czjzek, 1848)

Stainforthia fusiformis (Williamson, 1858)

Uvigerina peregrina Cushman, 1923

B. Species, which are considered either arctic or eurythermal:

Angulogerina fluens Todd, 1947

Astrononion gallowayi Loeblich and Tappan, 1953

Buccella frigida (Cushman, 1922)

Cassidulina reniforme Nørvang, 1945

Cibicides lobatulus (Walker and Jacob, 1798) 
Cibicides pseudoungerianus (Cushman, 1922)

Elphidium asklundi Brotzen, 1943

Elphidium bartletti Cushman, 1933

Elphidium excavatum (Terquem, 1875)

Elphidium groenlandicum Cushman, 1933

Elphidium hallandense Brotzen, 1943

Fissurina danica (Madsen, 1895)

Fissurina laevigata Reuss, 1850

Islandiella helenae Feyling-Hanssen and Buzas, 1976

Islandiella norcrossi (Cushman, 1933)

Lagena striata (d'Orbigny, 1839), forma typica

Miliolinella subrotunda (Montagu, 1803)

Nonion labradoricum (Dawson, 1960)

Nonion orbiculare (Brady, 1881)

Parafissurina lateralis (Cushman), forma carinata

(Buchner, 1940)

Pyrgo williamsoni (Silvestri, 1923)

Quinqueloculina seminulum (Linné, 1758)

Stainforthia loeblichi (Feyling-Hanssen, 1954)

\section{Summary and conclusions}

Foraminiferal faunas from a continuous marine sequence, reaching from Late Saalian through Eemian and Early Weichselian into the Middle Weichselian, have been described from the Apholm boring at Frederikshavn. The marine sequence, which rests on a non-marine Quaternary deposit, is subdivided into four foraminiferal zones, zone AP4 to zone AP1 (figs. 3a and $3 b$ ).

The lowermost zone $A P 4$, from about $190 \mathrm{~m}$ depth in the borehole, contains mainly arctic foraminifera. The zone is correlated with zone N4 of the Skærumhede I boring and with oxygen isotope stage 6 , and the faunal composition indicates that marine conditions first reached this area in Late Saalian times.

The faunas in AP3 indicate boreal to boreallusitanian conditions and water depths of at least $60-100 \mathrm{~m}$. This zone is correlatable with zone N3 of the Skxrumhede $I$ boring and with oxygen isotope stage $5 \mathrm{e}$, and it is referred to the Eemian Interglacial. Corresponding deep water facies of the Eemian have also been found in a series of other borings in North Jutland. They are known from Anholt (Lykke-Andersen, in prep.), western Norway and from the northern North Sea. Shallower and coastal facies of the Eemian are known from many localities farther to the south in Jutland and from the Baltic and southern North Sea areas.

The boreal to boreal-arctic zone $A P 2$ is correlated with zone N2 from the Skærumhede I boring and with zones IX and VIII of Skærumhede II. The faunas in zone AP2 reflect gradually colder conditions. The zone is placed in the Early Weichselian and correlated with oxygen isotope stages 5a-d. The foraminiferal faunas show that a considerable drop in sea-level took place at the transition from the Eemian (zone AP3) to the Weichselian (zone AP2). The faunal indications suggest, that this change in water depth may have been even larger than the $50 \mathrm{~m}$ suggested by Kellogg et al. (1978). Local tectonic effects cannot however, be overlooked in this area.

The zone AP3-AP2 boundary in Apholm is here correlated with the Eemian-Weichselian boundary. This boundary has earlier been placed higher in the sequence by e.g. Bahnson et al. (1974) and Knudsen (1976), who correlated the zone VIII-VII boundary and the base of the Portlandia arctica zone at Skærumhede with the Eemian-Weichselian boundary. Later Knudsen and Lykke-Andersen (1982) found evidence for a deeper position, and they tentatively proposed a correlation of the zone N3-N2 boundary with the transition from the Eemian to the Weichselian. This suggestion is supported by the present study.

The foraminifera of zone AP1 in Apholm indicate fluctuating high-arctic and boreal-arctic conditions. This zone is correlated with the lower part of zone N1 and Skærumhede I and with zones VII to IV in Skærumhede II. Zone AP1 represents the continuation of marine conditions in the area into the Middle Weichselian and is tentatively correlated with oxygen isotope stage 4 and a part of stage 3 . The uppermost boundary of the marine sequence at Apholm cannot however, be defined on the basis of the available information. It must be emphasized that the marine sequence contains no indication of any glaciations in the present area during the Early and Middle Weichselian times.

Acknowledgments. I am grateful to the Geological Survey of Denmark for placing samples from Apholm at my disposal. My thanks to Rolf W. Feyling-Hanssen and Anne-Lise LykkeAndersen for valuable discussions during the work, and to David N. Penney, University of Dublin, Ireland for improving the English of the manuscript. I would also like to thank Svend 
Meldgaard, who processed the samples, Jette Gissel Nielsen for making the drawings and Lissi $\emptyset_{\text {sterhaab Mogensen for typing }}$ the manuscript.

\section{Dansk sammendrag}

En marin Kvartær lagserie på mere end $100 \mathrm{~m}$ tykkelse $\mathrm{i}$ boringen Apholm 7.131b nord for Frederikshavn er foraminiferstratigrafisk undersøgt. Da materialet fra Apholm består af en serie uforstyrrede kerneprøver, giver unders $ø$ gelsen et værdifuldt supplement til tidligere foraminifer-stratigrafiske unders $\emptyset$ gelser fra området, nemlig Skærumhede I og II boringerne vest for Frederikshavn.

Den marine lagserie i Apholm, som hviler på en ikke-marin Kvartær aflejring, kan inddeles i 4 foraminifer-zoner. Zone AP4 (190,2 m dybde), der overvejende indeholder arktiske foraminiferer, kan korreleres med zone N4 i Skærumhede I og henføres til Sen Saale. Faunaerne i zone AP3 (190-140 m) tyder på temperaturer lidt højere end $i$ området $i$ dag og vanddybder på mindst $60-100 \mathrm{~m}$. Et indhold af planktoniske foraminiferer i enkelte niveauer tyder på en ret åben forbindelse til Norskehavet. Zone AP3 svarer nøje til zone N3 i Skærumbede I. Den formodes at repræsentere oxygen isotop enheden $5 \mathrm{e}$ og henføres til Eem Interglacial tid.

I zone AP2 (140-113 m) viser faunaerne en gradvis forværring af klimaet, idet arktiske arter dukker op. Vanddybden falder drastisk ved overgangen til zone AP2, men forøges atter en smule henimod slutningen af zone AP2. Zone AP2 kan korreleres med zone N2 i Skærumhede I og med zonerne IX og VIII i Skærumhede II. Den menes at reprasentere oxygen isotop enhederne 5a-d og henføres til en klimatisk overgangsfase i Tidlig Weichsel. Den pludselige ændring til lavere vanddybde ved grænsen mellem zonerne AP3 og AP2 svarer nogenlunde til et generelt fald $i$ havniveau på omkring $50 \mathrm{~m}$, som er beskrevet fra grænsen Eem-Weichsel. Muligvis kan også lokale tektoniske bevægelser have haft indflydelse.

Grænsen mellem zonerne AP3 og AP2 i Apholm er således her korreleret med Eem-Weichsel grænsen. Denne granse har tidligere været placeret højere i den marine lagserie, bl.a. af Bahnson et al. (1974) og Knudsen (1976), som foreslog græensen mellem foraminifer-zonerne VIII og VII, samt basis af Portlandica arctica zonen i Skærumhede korreleret med Eem-Weichsel grænsen. Senere unders øgelser af materiale fra Skærumhede I boringen, samt nye dateringer fra Hirtshals Kystklint, førte til, at Knudsen og Lykke-Andersen (1982) foreslog en placering af Eem-Weichsel grænsen dybere i lagserien, muligvis ved grænsen mellem foraminifer-zonerne N3 og N2, d.v.s. midt i Turritella terebra zonen. Denne antagelse støttes af unders $\emptyset$ gelserne fra Apholm.

Faunaerne i zone APl (113 m til øverste prøve fra $86 \mathrm{~m}) \mathrm{i}$ Apholm er hovedsagelig arktiske, men et skiftende borealt indhold tyder på, at 2 stadiale og 2 interstadiale perioder er repræsenterede. Som helhed kan zonen korreleres med en del af zone N1 i Skærumhede I. En nøjere sammenligning med foraminifer-zonerne i Skærumhede II viser, at underzonen AP1b kan omfatte zonerne VII, VI og V, mens underzonen AP1a sandsynligvis svarer til zone IV i Skærumhede II. Zone AP1 henføres til Weichsel, og synes at representere oxygen isotop enhed $4 \mathrm{og}$ en del af enhed 3.

De foraminifer-stratigrafiske unders $\emptyset$ gelser fra Apholm viser, at der har været en kontinuerlig marin sedimentation i området fra Sen Saale, gennem Eem og Tidlig Weichsel og et stykke ind i Mellem Weichsel. Den øvre grænse for den marine lagserie kan ikke fastlægges på det foreliggende materiale fra Apholm boringen, men det må imidlertid bemærkes, at den undersøgte lagserie fra Apholm ikke viser tegn på glaciationer i området, hverken i Tidlig eller i Mellem Weichsel.

\section{References}

Bahnson, H., Petersen, K. S., Konradi, P. B. and Knudsen, K. L. 1974: Stratigraphy of Quaternary deposits in the Skærumhede II boring: lithology, molluscs and foraminifera. Danm. geol. Unders., Arbog 1973, $27-62$.

Belanger, P. E. 1982: Paleo-oceanography of the Norwegian Sea during the past 130,000 years: coccolithophorid and foraminiferal data. Boreas, 11, 29-36.

Buzas, M. A. 1965: Foraminifera from Late Pleistocene clay near Waterville, Maine. Smithsonian Misc. Coll., 145 (8), 1-30.

Feyling-Hanssen, R. W. 1981: Foraminiferal indication of Eemian interglacial in the northern North Sea. Bull. geol. Soc. Denmark, 29, 175-189.

Feyling-Hanssen, R. W. 1982: Foraminiferal zonation of a boring in Quaternary deposits of the northern North Sea. Bull. geol. Soc. Denmark, 31, 29-47.

Feyling-Hanssen, R. W., Jørgensen, J. A., Knudsen, K. L. and Lykke-Andersen, A.-L. 1971: Late Quaternary Foraminifera from Vendsyssel, Denmark and Sandnes, Norway. Bull. geol. Soc. Denmark, 21 (2-3), 67-317.

Gevirtz, J. L., Park, R. A. and Friedman, G. M. 1971: Paraecology of benthonic foraminifera and of associated microorganisms of the continental shelf off Long Island, New York. J. Paleontol., 45, 153-177.

Giunta, M. 1954: Quinqueloculina pentagona n.sp. Riv. Ital. Pal. Strat., Milano, 60, no. 1, 33.

Hedberg, H. D. ed. 1976: International stratigraphic guide. A guide to stratigraphic classification, terminology and procedure. Wiley and Sons, New York, 1-200.

Höglund, H. 1947: Foraminifera in the Gullmar Fjord and the Skagerak. Zool. Bidr. frán Uppsala, 26, 1-328.

Jarke, J. 1961: Die Beziehungen zwischen hydrographischen Verhältnissen, Faziesentwicklung und Foraminiferenverbreitung in der heutigen Nordsee als Vorbild für die Verhältnisse während der Miocän-Zeit. Meyniana, 10, 21-36.

Jelgersma, S. 1979: Sea-level changes in the North Sea basin, 233-248. In: Oele, E., Schüttenhelm, R. T. E. and Wiggers, A. J. (eds.): The Quaternary history of the North Sea. Acta Univ. Ups. Symp. Univ. Ups. Annum Quing. Cel. 2, Uppsala, 1-248.

Jessen, A., Milthers, V., Nordmann, V., Hartz, N. and Hesselbo, A. 1910: En boring gennem de kvartære Lag ved Skærumhede. Danm. geol. Unders., II Rakke, 25, 1-175.

Kellogg, T. B. 1980: Paleoclimatology and paleo-oceanography of the Norwegian and Greenland seas: glacial-interglacial contrasts. Boreas, 9, 115-137.

Kellogg, T. B., Duplessy, J. L. and Shackleton, N. J. 1978: Planktonic foraminiferal and oxygen stratigraphy and paleoclimatology of Norwegian Sea deep-sea cores. Boreas, 7 , 61-73.

Knudsen, K. L. 1976: Foraminifer faunas in Weichselian stadial and interstadial deposits of the Skarumhede boring, Jutland, Denmark. Maritime sediments, Spec. Publ. $1, \mathrm{~B}-\mathrm{Pa}$ leoecology and Biostratigraphy, Halifax, 431-449.

Knudsen, K. L. 1984: Correlation of Saalian, Eemian and Weichselian foraminiferal zones in North Jutland. Bull. geol. Soc. Denmark, in press.

Knudsen, K. L. and Lykke-Andersen, A.-L. 1982: Foraminifera in Late Saalian, Eemian, Early and Middle Weichselian of the Skærumhede I boring. Bull. geol. Soc. Denmark, 30, 97-109.

Konradi, P. B. and Knudsen, K. L. 1974: Foraminifera in the Skærumhede II boring, 43-57. In: Bahnson, H., Petersen, K. S., Konradi, P. B. and Knudsen, K. L. 1974.

Lange, W. 1956: Grundproben aus Skagerrak und Kattegat, mikro-faunistisch und sedimentpetrographisch untersucht. Meyniana, 5, 51-86. 
Lykke-Andersen, A.-L. 1971: Foraminifera from the Older Yoldia Clay in Hirtshals, 159-184. In: Feyling-Hanssen, R. W., Jørgensen, J. A., Knudsen, K. L. and Lykke-Andersen, A.-L. Bull. geol. Soc. Denmark, 21 (2-3), 67-317.

Lykke-Andersen, A.-L. 1981: En ny C-14 datering fra Aldre Yoldia Ler i Hirtshals Kystklint. Dansk geol. Foren. Arsskrift for 1980, 1-5.

Lykke-Andersen, A.-L. 1982: Nogle nye C-14 dateringer fra Ældre Yoldia Ler i Hirtshals Kystklint. Dansk geol. Foren. Arsskrift for 1981, 119-121.

Mangerud, J., Sønstegaard, E. and Sejrup, H.-P. 1979: Correlation of the Eemian (interglacial) Stage and the deepsea oxygen-isotope stratigraphy. Nature, 277, 189-192.

Mangerud, J., Sønstegaard, E., Sejrup, H.-P. and Haldorsen, S. 1981: A continuous Eemian-Early Weichselian sequence containing pollen and marine fossils at Fjøsanger, western Norway. Boreas, 10, 137-208.

Meldgaard, S. and Knudsen, K. L. 1979: Metoder til indsamling og oparbejdning af prøver til foraminiferanalyser. Dansk Natur - Dansk Skole, Arsskrift 1979, 48-57.

Murray, J. W. 1973: Distribution and Ecology of Living Benthic Foraminiferids. Heinemann, London, 1-274.

Nagy, J. 1965: Foraminifera in some bottom samples from shallow waters in Vestspitsbergen. Norsk Polarinst. Arbok 1963, 109-125.

Risdal, D. 1964: Foraminiferfaunaenes relasjon til dybdeforholdene i Oslofjorden, med en diskusjon av de senkvartare foraminifersoner. Norges geol. Unders., 226, 1-142.

Ruddiman, W. F., McIntyre, A., Niebler-Hunt, V. and Durazzi, J. T. 1980: Oceanic evidence for the mechanism of rapid northern hemisphere glaciation. Quaternary Research, 13, 33-64.
Shackleton, N. J. and Opdyke, W. D. 1973: Oxygen isotope and paleomagnetic stratigraphy of equatorial Pacific core V28-238: Oxygen isotope temperatures and ice volumes on a $10^{5}$ year and $10^{6}$ year scale. Quatemary Research, 3, 39-55.

Streeter, S. S., Belanger, P. E., Kellogg, B. and Duplessy, J. C. 1982: Late Pleistocene Paleo-Oceanography of the Norwegian-Greenland Sea: Benthic Foraminiferal Evidence. Quaternary Research, 18, 72-90.

Thiede, J., Qvale, G., Skarboe, O. and Strand, J. E. 1981: Benthonic foraminiferal distributions in a southern Norwegian fjord system: a re-evaluation of Oslo Fjord data. Spec. Publs int. Ass. Sediment., 5, 469-495.

Vogel, J. C. 1982: Ionium dating of the Early Würm in Europe. XI INQUA Congress, Moscow, Abstracs II, 349.

Walton, W. R. 1964: Recent foraminiferal ecology and paleoecology. In: Imbrie, J. and Newell, N. D. (editors): $A p$ proaches to Paleontology, 151-237. Wiley and Sons, New York.

Weering, T. C. E. van and Qvale, G. 1983: Recent sediments and foraminiferal distribution in the Skagerrak, northeastern North Sea. Marine Geology, 52, 75-99.

Zagwijn, W. H. 1977: Sea level changes during the Eemian in the Netherlands. Xth INQUA Congress, Birmingham 1977, Abstracts, 509.

Zagwijn, W. H. 1983: Sea-level changes in the Netherlands during the Eemian, 437-450. In: Berg, M. W. van den and Felix, R. (eds.): Special issue in the honour of J. D. de Jong. Geol. Mijnbouw, 62. 